\title{
Acoustic reflection from the boundary of anisotropic thermoviscoelastic solid with fluid
}

\author{
M D SHARMA \\ Department of Mathematics, Kurukshetra University 136119 \\ e-mail: mohan_here@ rediffmail.com
}

MS received 10 December 2008; revised 4 May 2009

\begin{abstract}
Vertical slownesses of waves at a boundary of an anisotropic thermoviscoelastic medium are calculated as roots of a polynomial equation of degree eight. Out of the corresponding eight waves, the four, which travel towards the boundary are identified as upgoing waves. Remaining four waves travel away from the boundary and are termed as downgoing waves. Reflection and refraction of plane harmonic acoustic waves are studied at a plane boundary between anisotropic thermoviscoelastic solid and a non-viscous fluid. At this fluid-solid interface, an incident acoustic wave through the fluid reflects back as an attenuated acoustic wave and refracts as four attenuating waves into the anisotropic base. Slowness vectors of all the waves in two media differ only in vertical components. Complex values of vertical slowness define inhomogeneous refracted waves with a fixed direction of attenuation, i.e. perpendicular to the interface.

Energy partition is calculated at the interface to find energy shares of reflected and refracted waves. A part of incident energy dissipates due to interaction among the attenuated refracted waves. Numerical examples are considered to study the variations in energy shares with the direction of incident wave. For each incidence, the conservation of incident energy is verified in the presence of interaction energy. Energy partition at the interface seems to be changing very slightly with the azimuthal variations of the incident direction. Effects of anisotropy, elastic relaxation and thermal parameters on the variations in energy partition are discussed. The acoustic wave reflected from isothermal interface is much significant for incidence around some critical directions, which are analogous to the critical angles in a non-dissipative medium. The changes in thermal relaxation times and uniform temperature of the thermoviscoelastic medium do not show any significant effect on the reflected energy.
\end{abstract}

Keywords. Anisotropy; thermoviscoelastic; acoustic waves; reflection; attenuation.

\section{Introduction}

Every man-made material on our planet undergoes appreciable change of volume when subjected to the variation in temperature. Conversely, to a change in volume, an elastic medium 
responds with a change of temperature. Thermoelasticity deals with the dynamical systems whose interactions with the surroundings include not only mechanical work and external work but the exchange of heat also. In the earlier references, thermodynamics of an elastic solid is found in a study by Jeffereys (1930). After that, Biot (1956) explained thermoelasticity by deriving dilatation based on the thermodynamics of irreversible process and coupling it with elastic deformation. But, the diffusion type heat equation used in this study predicted an infinite speed for propagation of temperature. Lord \& Shulman (1967) defined the generalized theory of thermoelasticity (termed as LS theory) in which a hyperbolic equation for heat conduction with a relaxation time ensured a finite speed for thermal signals. Another generalization (called GL theory) with an additional relaxation time to represent the effect of time rate of temperature change on elastic disturbance came from Green \& Lindsay (1972). From Puri (1973) to Sharma et al (2003), a large number of problems has been studied on the propagation of plane waves in generalized thermoelastic media. In the intervening years, the linear theories of generalized thermoelasticity have been modified to avoid energy-dissipation (Green \& Naghadi 1993). Hetnarski \& Ignaczak (1996) deviated from the linear convention and introduced low temperature thermoelasticity with non-linear flow of elastic heat. Giorgi et al (2001) studied a linear theory for thermoviscoelastic materials but with thermal behaviour represented through the heat conduction equation. El-Karamany et al (2002) and El-Karamany \& Ezzat (2002) described and established relaxation effects in mechanical properties as well as thermo-mechanical coupling in thermoviscoelastic media.

Isotropy exists only for our convenience. Any real material may be assumed anisotropic unless and until it has been shown that it is sufficiently close to our assumption of isotropy. Musgrave (1970) studied stress waves in crystals by treating them as anisotropic continua. A book by Carcione (2001) explains the importance of anisotropy for wave propagation studies in real materials. Dhaliwal \& Sheriff (1980) modified the LS theory of thermoelasticity for anisotropic materials. Singh \& Sharma (1985) discussed the wave propagation in transversely isotropic generalized thermoelastic medium. Sharma et al (2000) studied the propagation of plane harmonic waves in orthotropic thermoelastic materials. In recent studies (Sharma 2006, 2007), the propagation of homogeneous and inhomogeneous waves are discussed in thermoelastic media with arbitrary anisotropy. In another study (Sharma 2008), the present author solved the Christoffel equations for anisotropic thermoelastic medium into an eighthdegree polynomial in vertical slowness. These eight slowness values explain the propagation of eight attenuating waves at the boundary of the medium. The propagation characteristics of the downgoing (decaying away from the boundary) waves were also discussed.

Coexistence of thermoelasticity and anisotropy is a common happening in the most of the industrial phenomena. Polymer composites are a wide class of synthetic materials having a wide range of mechanical and thermal properties. The fibre-reinforced polymer composites are manufactured as shock absorbing materials. Anelastic relaxation is considered in crystals also (Norwick \& Berry 1972). Both polymers and crystals are rheologically characterized as anisotropic viscoelastic materials. Thermally conducting polymer composites are used as packaging materials to meet the heat dissipation requirements in various industrial phenomena. Thermal and mechanical properties of some polymer composites were determined in a study by Wong \& Bollampally (1999). The thermoviscoelastic analysis of polymers is important for exploring their sound or shock absorbing properties. This requires an insight into the effects of strain rate, temperature sensitivities and thermo-mechanical coupling on the deformation behaviour of polymers. The present study considers the problem of reflection and refraction at a plane interface between non-viscous fluid and anisotropic thermoviscoelastic (referred as ATV, hereafter) medium. An acoustic wave travels through the fluid and is 
incident at this interface. The resulting reflected acoustic wave is studied through its complex amplitude, which is related to its energy share and phase shift. The part of energy refracted to the dissipative ATV medium is separated as the refraction coefficients of four attenuated waves and interaction energy among them. Numerical examples are computed to analyse the effects of thermoelasticity theory (LS or GL) and elastic and thermal properties of ATV medium on energy partition at the boundary of alumina-epoxy with water.

\section{Definition of the problem}

An acoustic wave propagating through an inviscid fluid is incident at its plane interface with anisotropic thermoviscoelastic (ATV) medium. A part of the incident energy reflects back as an acoustic wave and the remaining energy refracts into the continuing ATV medium and propagates as four attenuating waves. The procedure to study the propagation of reflected and refracted waves is explained as follows.

(i) For propagation of plane harmonic waves in ATV medium, the resulting Christoffel equations are solved into a polynomial equation of degree eight. Numerically obtained eight roots of this equation define the vertical slowness values for four upgoing and four downgoing waves in the medium.

(ii) The four downgoing waves, which satisfy decay condition in the semi-infinite medium, are identified as the refracted waves.

(iii) The slowness vectors of these four downgoing waves are used to define the displacement of particles in ATV half-space.

(iv) At isothermal (or thermally insulated) interface, reflection-refraction phenomena is studied to calculate the distribution of incident energy among reflected and refracted waves.

(v) Numerical examples are considered to compute the energy share and phase shift of lone reflected wave. Energy shares of the attenuating refracted waves and interaction energy among them are calculated to verify the conservation of energy at the interface.

\section{Anisotropic thermoviscoelastic (ATV) medium}

A homogeneous anisotropic thermally conducting elastic solid is considered at a uniform temperature $T_{o}$ in the undisturbed state. In the absence of body forces and thermal sources, the propagation phenomenon (Sharma 2006) of plane harmonic waves in the anisotropic thermoelastic medium is explained through two relations. One of them, given by

$$
T=\imath \omega \frac{T_{o}\left(\tau_{0} \delta_{1 m}+\imath / \omega\right)}{K_{j k} p_{j} p_{k}-\rho C_{e}\left(\tau_{0}+\imath / \omega\right)} \alpha_{j k} p_{k} u_{j},
$$

relates the displacement ( $\mathbf{u})$ of particles to temperature change $(T)$ in the medium. Here, $\delta_{j m}$ is Kronecker delta so that, $m=1$ represents Lord-Shulman (LS) and $m=2$ represents Green-Lindsay (GL) theory of thermoelasticity. The dummy index implies summation. The unknowns $\left(u_{j}\right.$ and $\left.T\right)$ are expressed as

$$
\begin{aligned}
u_{j} & =S_{j} \exp \left\{\imath \omega\left(p_{k} x_{k}-t\right)\right\} ; \quad(j=1,2,3), \\
T & =\Theta \exp \left\{\imath \omega\left(p_{k} x_{k}-t\right)\right\},
\end{aligned}
$$


where, components $\left(S_{1}, S_{2}, S_{3}\right)$ define the polarization vector $\mathbf{S}$ for the displacement of the particles in the medium. The components $\left(p_{1}, p_{2}, p_{3}\right)$ define slowness vector $\mathbf{p}$ for wave propagation in the medium.

The other relation represents the modified Christoffel equations, given by

$$
W_{i k} S_{k}=0 ; \quad W_{i k}=-\rho \delta_{i k}+c_{i j k l} p_{j} p_{l}-T_{o} \tau^{\prime} \frac{\alpha_{i j} p_{j} \alpha_{k l} p_{l}}{K_{j l} p_{j} p_{l}-\rho C_{e} \tau}
$$

where, $c_{i j k l}$ are isothermic elastic constants and the positive-definite tensor $K_{i j}$ denotes thermal conductivity. The non-singular tensor $\alpha_{i j}$ represents thermoelastic coupling. The $\rho$ and $C_{e}$ denote density and specific heat at constant strain, respectively. The complex time parameters $\tau=\tau_{0}+\imath / \omega$ and $\tau^{\prime}=\tau_{0} \delta_{1 m}+\tau_{1} \delta_{2 m}+\imath / \omega$ involve circular frequency $\omega$ and two thermal relaxation times (i.e. $\tau_{0} \geq \tau_{1}$ ).

The elastic-viscoelastic correspondence principle remains valid for thermoelastic medium also (Kovalenko \& Karnaukhov 1972; Paulino \& Jin 2001). This implies that the linear viscoelasticity in a thermoelastic medium is represented by the complex values of its elastic constants and thermo-mechanical coupling constants. Then, for propagation in ATV medium, the real elastic tensors $c_{i j k l}$ and $\alpha_{i j}$ are replaced with complex (dual) tensors.

A non-trivial solution of the Christoffel equations is ensured by a determinantal equation, given by

$$
\operatorname{det} \mathbf{W}=0
$$

For incidence of a wave at the boundary of ATV medium, Snell's law (Helbig 1994) implies that the values of $p_{1}, p_{2}$ for the incident wave remain unchanged for all the resulting waves on either side of the boundary. Hence, to solve the system (3), assume that $p_{1}, p_{2}$ are known and vertical slowness $p_{3}$ is the only unknown. For notational simplicity in the analytical calculations, unknown $p_{3}$ is replaced by $q$. In terms of $q$, the matrix $\mathbf{W}$ in (3) is resolved as

$$
\mathbf{W}=\mathbf{A} q^{2}+\mathbf{B} q+\mathbf{C}+\frac{1}{q^{2}+a q+b}\left(\mathbf{D}^{\prime} q+\mathbf{E}^{\prime}\right),
$$

where the scalars $a, b$ and the coefficient matrices $\left(\mathbf{A}, \mathbf{B}, \mathbf{C}, \mathbf{D}^{\prime}, \mathbf{E}^{\prime}\right)$ are the same as expressed in Sharma (2008). Then the determinantal equation (4) is solved into a polynomial equation of degree eight, given by

$$
\sum_{j=0}^{8} C_{j} q^{j}=0 .
$$

The coefficients $C_{j}$ are defined in Sharma (2008). This equation is solved for eight values of $q$, with the given values of $p_{1}$ and $p_{2}$. With same $\left(p_{1}, p_{2}\right)$ for all the waves in ATV medium, different waves are identified, mathematically, with their vertical slowness $(q)$ values. The complex slowness vector $\left(p_{1}, p_{2}, q\right)$ of a wave is used to calculate its polarization from the system of equations (3). The existence and propagation of four attenuating waves in an infinite anisotropic thermoelastic medium has been explained in an earlier study (Sharma 2007). These were identified as a quasi-longitudinal wave, two quasi-transverse waves and a quasi-thermal wave. The prefix 'quasi' is used to indicate that these waves are not exactly longitudinal $(P)$ or transverse $(S)$ in character. With descending velocities along a given phase direction, these waves may be identified as $q P, q S 1, q S 2, q T$ waves. So, the eight complex values of vertical slowness identify the propagation of four attenuating waves as either decaying upward (positive $x_{3}$-direction) or downward (negative $x_{3}$-direction) in the medium. 


\section{Reflection and refraction}

The eight roots of equation (6) explain the upward and downward propagation of four attenuating waves in the ATV medium. These roots (i.e. $q$ s) define the slowness vector $\left(p_{1}, p_{2}, q\right)$ for each of the eight waves existing at the boundary of the medium. For propagation in a layer, all the eight waves contribute to the total disturbance in the medium. But, for propagation in a semi-infinite medium (or half-space) only four (downgoing) waves are significant.

\subsection{Geometry of the medium}

Consider a fluid half-space in contact with ATV half-space along a plane interface. In rectangular Cartesian coordinate system $\left(x_{1}, x_{2}, x_{3}\right)$, the plane $x_{3}=0$ represents a common interface between the two media and the $x_{3}$-axis is pointing upward into the fluid medium. An acoustic wave travel through the fluid and become incident at a point on the interface. In a local spherical co-ordinate system centered at this point, let $(\theta, \phi)$ be a general direction in the medium with $\theta$ as polar angle with the $x_{3}$-direction and azimuth $\phi$ is measured from $x_{1}$-axis to $x_{2}$-axis. This incident wave in fluid reflects back as an acoustic wave and refracts to ATV medium as four attenuating waves. A direction $\left(\theta_{o}, \phi_{o}\right)$ of incident acoustic wave in fluid defines its slowness vector $\mathbf{p}=\left(\sin \theta_{o} \cos \phi_{o}, \sin \theta_{o} \sin \phi_{o}, \cos \theta_{o}\right) / v_{o}$, where $v_{o}$ denotes the velocity of incident wave. Then according to Snell's law, the vertical plane $\phi=\phi_{o}$ contains the phase directions of all the reflected and refracted waves at the interface. Thus, a vertical plane with (arbitrarily) fixed $\phi_{o}$ may be defined as the propagation plane in which $\theta_{o}$ specifies the direction of the incident wave.

\subsection{Slownesses of refracted waves}

The values of $p_{1}$ and $p_{2}$ for the incident wave are used to calculate eight values of $q$ from (6). Out of the eight, only four correspond to downgoing refracted waves resulting from the incidence of acoustic wave. For a complex vertical slowness value, the sign of its imaginary part decides whether a wave is upgoing or downgoing (refracted) wave. In fact, a downgoing wave in the medium must attenuate (decay) on moving away from the boundary. In the present geometry, a vertical slowness value with positive imaginary part represents a refracted wave. It is, generally, expected that there should be four such slowness values $\left(q_{j}, j=1,2,3,4\right)$ for each angle of incidence. The propagation part $\left(p_{1}, p_{2}, \Re q\right)$ of slowness vector defines the propagation direction and (inverse of) phase velocity of the corresponding refracted wave. Due to the incidence through a loss-less fluid medium the attenuation vectors of refracted waves are always perpendicular to the interface. This implies that the refracted waves are inhomogeneous waves with inhomogeneity angle equal to propagation angle. The quality factors of attenuation $(Q)$ of these inhomogeneous waves are defined by $Q^{-1}=\Im\left(q^{2}\right)$ / $\left(p_{1}^{2}+p_{2}^{2}+\Re\left(q^{2}\right)\right)$.

\subsection{Displacements and stresses}

Displacement components in the fluid medium are written as

$$
u_{j}^{(o)}=n_{j} \exp \left\{l \omega\left(\frac{1}{v_{o}} n_{k} x_{k}-t\right)\right\}+a_{R} n_{j}^{\prime} \exp \left\{l \omega\left(\frac{1}{v_{o}} n_{k}^{\prime} x_{k}-t\right)\right\}, \quad(j=1,2,3),
$$


where $n_{1}=n^{\prime}{ }_{1}=\sin \theta_{o} \cos \phi_{o}, n_{2}=n_{2}^{\prime}=\sin \theta_{o} \sin \phi_{o}$ and $n_{3}=-n_{3}^{\prime}=\cos \theta_{o}$ define the phase directions of incident wave and reflected wave. In the fluid medium with density $\rho_{o}$ and acoustic velocity $v_{o}$, normal stress is given by

$$
\sigma_{33}^{(o)}=\rho_{o} v_{o}^{2} u_{j, j}^{(o)} .
$$

Displacement components and temperature variations in ATV solid half-space are expressed as

$$
\begin{gathered}
u_{j}=\sum_{r=1}^{4} f_{r} S_{j}^{(r)} \exp \left\{\imath \omega\left(q_{r} x_{3}+p_{1} x_{1}+p_{2} x_{2}-t\right)\right\}, \\
T=\sum_{r=1}^{4} f_{r} \Theta^{(r)} \exp \left\{\imath \omega\left(q_{r} x_{3}+p_{1} x_{1}+p_{2} x_{2}-t\right)\right\},
\end{gathered}
$$

where $f_{r}$ are relative excitation factors for the refracted waves. Constitutive relations for effective stresses in the medium are given by

$$
\sigma_{i j}=c_{i j k l} u_{k, l}-\alpha_{i j}\left(T+\tau_{1} \delta_{2 m} \dot{T}\right) .
$$

\subsection{Boundary conditions}

Boundary conditions come from the continuity of stress across the interface between fluid and ATV solid. The continuity of normal components of velocity is also considered. The thermally insulated (or isothermal) boundary is represented with the vanishing of $\partial T / \partial x_{3}$ (or $T$ ). So, in this study, the five appropriate boundary conditions to be satisfied at the plane $x_{3}=0$ are;

i) $\sigma_{13}=0$, ii) $\sigma_{23}=0$, iii) $\sigma_{33}=\sigma_{33}^{(o)}$, iv) $\psi T+(1-\psi) \frac{\partial T}{\partial x_{3}}=0$, v) $u_{3}=u_{3}^{(o)}$.

The parameter $\psi$ may be switched to define the boundary as thermally insulated (i.e. $\psi=0$ ) or isothermal (i.e. $\psi=1$ ). Satisfying these boundary conditions yields a system of five linear non-homogeneous equations in $f_{1}, f_{2}, f_{3}, f_{4}$ and $a_{R}\left(=f_{5}\right)$. These equations are given by

$$
a_{i j} f_{j}=b_{i} ; \quad(i=1,2,3,4,5),
$$

where, for column matrix $\mathbf{P}^{(\mathbf{j})}=\left(p_{1}, p_{2}, q_{j}\right)^{T}$, and row matrix $\mathbf{S}^{(\mathbf{j})}=\left(S_{1}^{(j)}, S_{2}^{(j)}, S_{3}^{(j)}\right)$, the coefficients $a_{i j}$ in (12) can be expressed as follows.

$$
\begin{aligned}
& a_{i j}=\mathbf{S}^{(j)} \mathbf{C}^{(i)} \mathbf{P}^{(j)}+\alpha_{i 3} \Theta^{(j)}\left(\tau_{1} \delta_{2 m}+\imath / \omega\right), \quad(i=1,2,3 ; j=1,2,3,4) ; \\
& a_{4 j}=\left[\psi+\imath \omega(1-\psi) q_{j}\right] \Theta^{(j)}, \quad a_{5 j}=S_{3}^{(j)}, \quad(j=1,2,3,4) ; \\
& a_{15}=a_{25}=b_{1}=b_{2}=0, \quad a_{35}=-b_{3}=-\rho_{o} v_{o}, \quad a_{45}=b_{4}=0 ; \quad a_{55}=b_{5}=q_{o} v_{o} .
\end{aligned}
$$

The third order square matrices $\mathbf{C}^{(i)}$ are defined as

$$
C^{(i)}=\left\{c_{l 1}, c_{l 6}, c_{l 5} ; c_{l 6}, c_{l 2}, c_{l 4} ; c_{l 5}, c_{l 4}, c_{l 3}\right\} ; \quad(l=6-i, \quad i=1,2,3),
$$

where $c_{I J}$ denotes the elastic tensor $c_{i j k l}$ in two-suffixed (Voigt) notations. 


\subsection{Energy partition}

Reflection (refraction) coefficient may be defined as the energy share of a reflected (refracted) wave in the energy of incident wave. Distribution of energy between reflected and different refracted waves is considered across a surface element of unit area at the plane interface $x_{3}=0$. The average energy flux of a wave across the interface, denoted by $\left\langle P^{*}\right\rangle$, is the time average of scalar product of its surface traction and particle velocity. With $x_{3}$-axis as the normal to the interface in the fluid medium, the average energy fluxes of incident wave and reflected wave are $\left\langle P_{I}^{*}\right\rangle=-0.5 \omega^{2} \rho_{o} v_{o} n_{3}$ and $\left\langle P_{R}^{*}\right\rangle=-0.5 \omega^{2}\left|a_{R}\right|^{2} \rho_{o} v_{o} n^{\prime}{ }_{3}$, respectively. The ratio $\left\langle P_{R}^{*}\right\rangle /\left\langle P_{I}^{*}\right\rangle$ defines the reflection coefficient of the reflected wave as $E_{R}=\left|a_{R}\right|^{2}$, where the omitted negative sign implies that the energy (or wave) is travelling in fluid but away from the reflecting boundary. The argument of complex amplitude $a_{R}$ defines the phase shift (denoted as $\phi_{R}$ ) of the reflected wave. Energy-amplitude relation for the reflected wave is given by

$$
a_{R}=\sqrt{E_{R}} e^{l \phi_{R}} .
$$

The variation of phase angle (or shift) with propagation direction implies the spatial dispersion of reflected waves. The dispersive phase behaviour of reflection coefficient is important for tracing of ray-paths.

The waves refracted across the interface propagate as inhomogeneous waves accompanied with interaction energy (Borcherdt 1977). It is a net energy flux across the interface which comes from the interaction of the velocity field of one wave with the stress field of another wave. This local energy flow does not result in any net radiation but is associated with net energy dissipation. It is significant for calculation of energy transmission coefficients and ensures the conservation of energy across the interface. To ensure energy conservation, Ainslie \& Burns (1995) have explained some derivations involving interference energy or complex energy ratios. In the present problem, an energy matrix $E_{j k}=\left\langle P_{j k}^{*}\right\rangle /\left\langle P_{I}^{*}\right\rangle ;(j, k=$ $1,2,3,4)$, is defined to explain the partition of energy refracted to the dissipative medium. The average energy fluxes in the ATV medium are defined as

$$
\left\langle P_{j k}\right\rangle=-0 \cdot 5 \omega^{2} \sum_{i=1}^{3} \Re\left[a_{i j} f_{j} \operatorname{conj}\left(S_{i}^{(k)} f_{k}\right)\right], \quad(j, k=1,2,3,4) .
$$

The sum of all the non-diagonal entries of the energy matrix $\left\{E_{j k}\right\}$ calculates the share of interaction energy (denoted with $E_{\text {int }}$ ). The diagonal entries of this matrix represent energy ratios of the four refracted waves in ATV medium. The conservation of energy is obtained through the relation $\sum_{j=1}^{4} \sum_{k=1}^{4} E_{j k}+E_{R}=1$. In terms of $E_{R}$ and $E_{\text {int }}$, the total energy share of four refracted inhomogeneous waves is, thus, given by $1-E_{\mathrm{int}}-E_{R}$.

\section{Numerical examples}

The procedure discussed in this study solves a general mathematical model for the reflection and refraction of plane harmonic waves at a plane boundary between fluid and ATV medium. The expressions derived in the study define the effects of various parameters on energy partition at the interface. These expressions are implicit and not very simple. Hence, the effects of various parameters can only be analysed through numerical examples. The physical model considered for numerical work is a plane surface of Alumina-filled epoxy submerged in water. 
The water of density $1000 \mathrm{~kg} / \mathrm{m}^{3}$ may allow sound to propagate with velocity $1463 \mathrm{~m} / \mathrm{s}$. The epoxy composite is considered an ATV medium, whose anisotropic (real) elastic constants (in $G P a$ ), following Wong \& Bollampally (1999), are chosen as

$$
\begin{aligned}
& c_{11}=5.1 \quad c_{12}=1.55 \quad c_{13}=1.45 \quad c_{14}=0.24 Z_{1} \quad c_{15}=-0.28 Z_{1} \quad c_{16}=0.32 Z_{2} ; \\
& c_{22}=5.8 \quad c_{23}=1.6 \quad c_{24}=-0.41 Z_{1} \quad c_{25}=-0.07 Z_{1} \quad c_{26}=1.13 Z_{2} ; \\
& c_{33}=5.4 \quad c_{34}=-0.12 Z_{1} \quad c_{35}=-0.01 Z_{1} \quad c_{36}=0.38 Z_{2} ; \\
& c_{44}=2.25 \quad c_{45}=-0.66 Z_{2} \quad c_{46}=0.06 Z_{1} \quad c_{55}=2.05 \quad c_{56}=0.52 Z_{1} \quad c_{66}=1.85 .
\end{aligned}
$$

A symmetric matrix $\boldsymbol{\Sigma}=\left\{1, .1 Z_{2}, .2 Z_{1} ; .1 Z_{2}, 1 \cdot 1, .15 Z_{1} ; .2 Z_{1}, .15 Z_{1}, .9\right\}$ is used to define the arbitrary anisotropy in thermal conductivity tensor $K_{i j}=\chi_{o} \Sigma_{i j}$ and thermoelastic coupling tensor $\alpha_{i j}=\alpha_{o}\left(3 c_{12}+2 c_{44}\right) \sum_{i j}$. The triclinic (or general) anisotropy (referred as, TCS) is defined with $Z_{1}=Z_{2}=1$. Then $Z_{1}=0$ defines the presence of monoclinic symmetry (MCS) and further $Z_{2}=0$ represents the orthotropic symmetry (ORS). The ratio $\tau_{1} / \tau_{0}=0 \cdot 1$ with $\tau_{0}=10^{-8} \mathrm{sec}$ fixes the two relaxation times. A fixed circular frequency of $100 \mathrm{kHz}$ is used to define $\omega$. The values for thermal parameters chosen for 30\% alumina-filled epoxy (Wong \& Bolampally 1999) are $\alpha_{o}=0.6 / 10^{4} \mathrm{~K}, \chi_{o}=0.6 \mathrm{~W} / \mathrm{mK}$ and $C_{e}=300 \mathrm{~J} / \mathrm{kgK}$. The boundary between water and alumina-epoxy is assumed isothermal with $T_{o}=300 \mathrm{~K}$.

The linear viscoelastic relaxation in the ATV medium is defined by multiplying the real elastic constants with complex factors. In this study, the constants $c_{11}, c_{22}, c_{33}$ are multiplied with $(1-0.03 \imath \epsilon)$. The constants $c_{44}, c_{55}, c_{66}$ are multiplied with $(1-0.01 \imath \epsilon)$ and $c_{12}, c_{21}, c_{13}, c_{31}, c_{23}, c_{32}$ are multiplied with $(1+0.03 \imath \epsilon)$. The complex factors are chosen so that, on reducing to isotropy, the viscoelastic tensors yield quality factor $Q_{P}=100$ and $Q_{S}=33.3$. The parameter $\epsilon$ is considered to vary the strength of viscoelasticity in the medium. In general, $\epsilon=1$ is used for numerical computations.

The above numerical values are used to calculate the quality factors of four attenuating waves refracted to ATV solid through its isothermal boundary with water. For triclinic anisotropy, these factors are obtained for incidence in a fixed vertical plane, $\phi=0 \cdot 34 \pi$. The variations of these attenuation factors with incident angle $\left(\theta_{o}\right)$ are exhibited in figure 1 . It is observed that near $\theta_{o}=53^{\circ}$, the real part of $q$ for refracted $q P$ wave changes sign. Due to this, the corresponding quality factor $(Q)$ becomes very large on either side of this angle. These large values are discarded to exhibit the variations for other directions. Hence, there is a break in the curve for $q P$ wave. This angle represents a critical incident direction beyond which refracted $q P$ wave in ATV medium changes direction and starts propagating towards the interface. This is analogous to the critical angle in a non-dissipative medium. In figure 1, the variations of $Q$ values for attenuated refracted waves ensure that the relaxation considered in the numerical example is realistic and anisotropic. The variations of $Q$ s were also computed with the azimuth $\left(\phi_{o}\right)$ of incident direction. These were found to be uniform and steady.

The main purpose of the numerical example is to calculate the energy partition at the isothermal surface of ATV model using the relations derived in previous sections. The values of $E_{R}$ and $\phi_{R}$ define the strength and phase variation of the reflected acoustic wave. The $E_{\mathrm{int}}$ alongwith $E_{R}$ determines the energy carried by the attenuated refracted waves and ensures the conservation of energy incident at the boundary. Figure 2 exhibits the variations of $E_{R}, \phi_{R}$ and $E_{\text {int }}$ with incident direction, for both the (LS and GL) theories of thermoelasticity. In threedimensional space, the incident directions are considered with $\left(\theta_{o}, \phi_{o}\right)$ varying from $(0,0)$ to $(\pi / 2, \pi / 2)$. Few important observations from the plots in this figure may be explained as follows. 

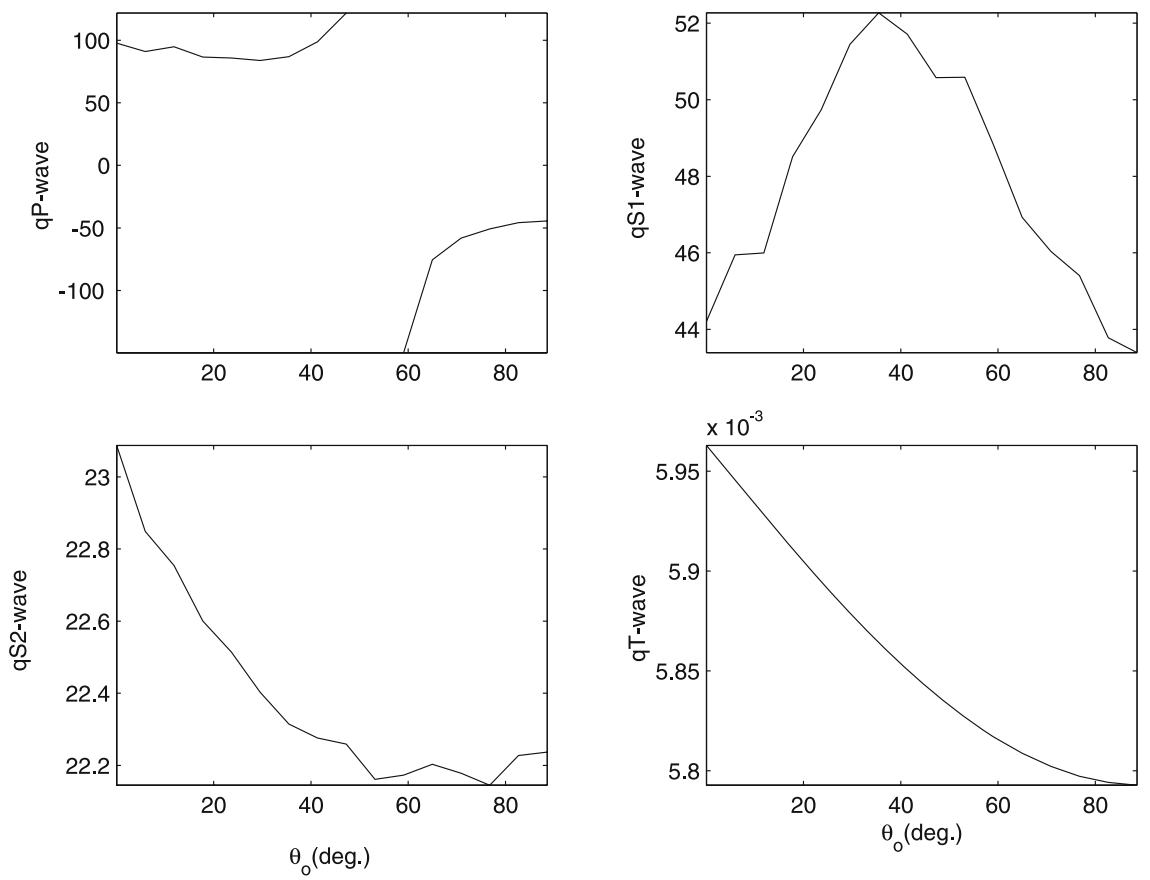

Figure 1. Variations of quality factors of attenuation $(Q)$ of four refracted waves with incident direction $\left(\theta_{o}\right)$ in $\phi_{o}=0.34 \pi$ plane, using GL theory of thermoelasticity in general anisotropic alumina-epoxy; $\left(\epsilon=1 ; \omega / 2 \pi=100 \mathrm{kHz} ; \tau_{0}=10^{-8} \mathrm{~s} ; T_{o}=300 \mathrm{~K} ; C_{e}=300 \mathrm{~J} / \mathrm{kgK} ; \chi_{o}=0.6 \mathrm{~W} / \mathrm{mK}\right.$; $\left.\alpha_{o}=0 \cdot 6 / 10^{4} \mathrm{~K}\right)$.

(i) The theory of thermoelasticity chosen makes only a negligible difference to the energy share of the reflected wave. The little difference observed is near the critical direction $\theta_{o}=53^{\circ}$. Significant changes in the phase shift and interaction energy are observed but only near this critical direction.

(ii) The plots are not changing much with the azimuth of incident direction (i.e. $\phi_{o}$ ). This implies that the propagation restricted to a vertical plane may be able to explain the effects of physical, elastic and thermal properties on the reflected wave.

(iii) In every vertical propagation plane, the variations of $E_{R}, \phi_{R}, E_{\text {int }}$ are much significant only for incidence beyond a particular angle. The polar angle (i.e. $\theta_{o}$ ) for this critical incident direction lies between $50^{\circ}$ to $60^{\circ}$.

(iv) The energy $\left(E_{R}\right)$ and the phase shift $\left(\phi_{R}\right)$ of reflected wave vary rapidly near grazing incidence. On the other hand, very slow and steady variations are observed when incidence is near-normal.

Taking clues from the above observations, the numerical calculation and analysis are restricted for incidence in a fixed vertical plane $\left(\phi_{o}=0 \cdot 34 \pi\right)$ using the GL theory of thermoelasticity only. The effects of medium properties on the reflected and refracted waves are discussed as follows.

\subsection{Anisotropy}

The energy share $\left(E_{R}\right)$ and phase shift $\left(\phi_{R}\right)$ of reflected wave and interaction energy among refracted waves $\left(E_{\text {int }}\right)$ are computed for three major anisotropies. Their variations with 

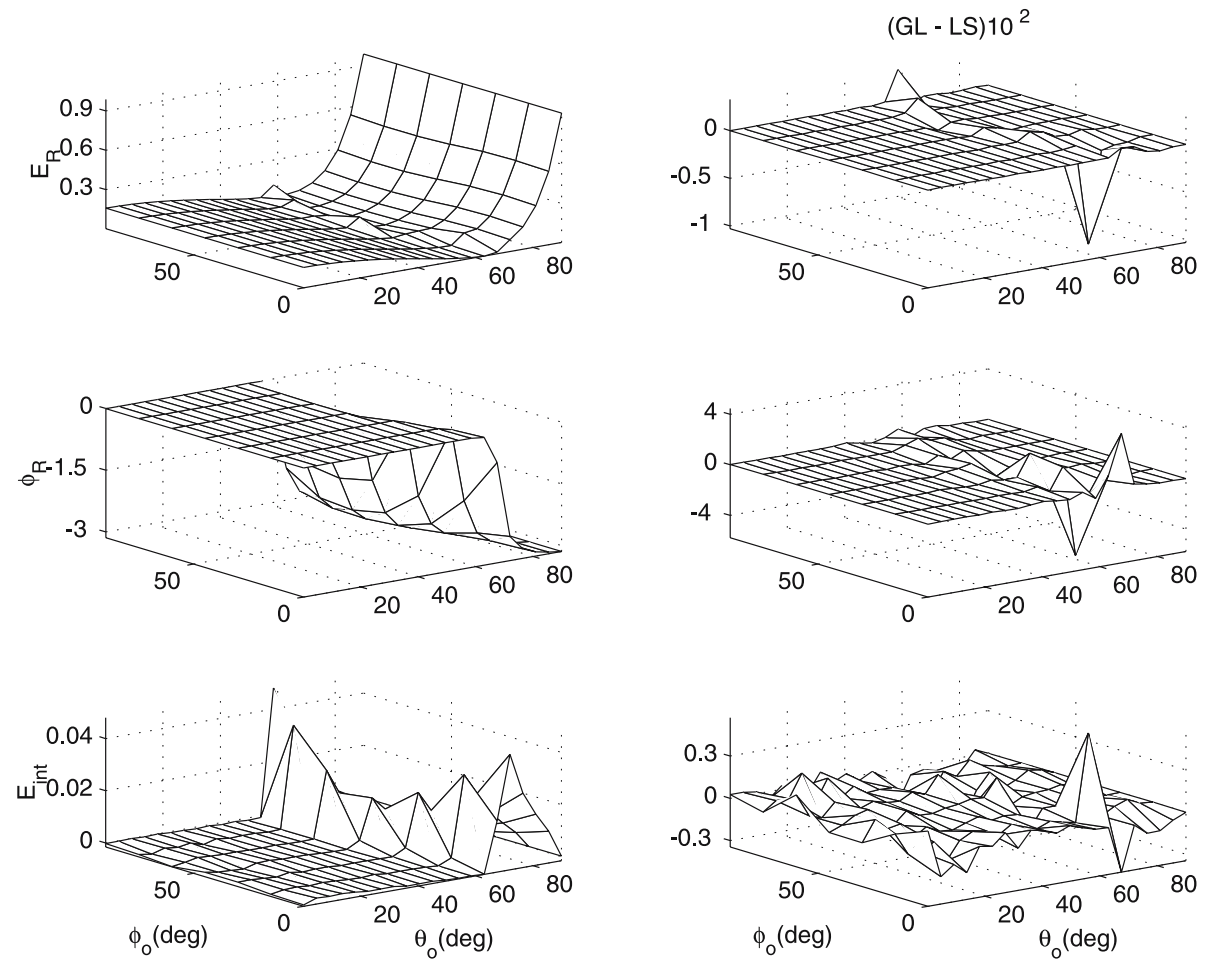

Figure 2. Variations of energy share $\left(E_{R}\right)$ and phase shift $\left(\phi_{R}\right)$ of reflected wave and interaction energy $\left(E_{\text {int }}\right)$ with incidence direction $\left(\theta_{o}, \phi_{o}\right)$ for the LS and the GL theories of thermoelasticity in general anisotropic alumina-epoxy; $\left(\epsilon=1 ; \omega / 2 \pi=100 \mathrm{kHz} ; \tau_{0}=10^{-8} \mathrm{~s} ; T_{o}=300 \mathrm{~K}\right.$; $\left.C_{e}=300 \mathrm{~J} / \mathrm{kgK} ; \chi_{o}=0.6 \mathrm{~W} / \mathrm{mK} ; \alpha_{o}=0.6 / 10^{4} \mathrm{~K}\right)$.

incident angle are shown in figure 3. The presence of monoclinic symmetry has almost no effect on the reflected wave as well as interaction energy. However, the presence of orthotropic symmetry has a noticeable effect on the phase shift of reflected wave and the interaction energy but only for incidence beyond $50^{\circ}$. The slight changes in reflected energy are also observed. It may be important to note that the critical incident direction for refracted $q P$ wave advances for the presence of orthotropic symmetry. Similar to the figure 2, the reflected energy attains the lowest value after this critical direction and then increases swiftly as the incidence goes to grazing.

\subsection{Anelasticity}

For the presence of anelasticity in thermoelastic solid bed, energy partition is computed for three different values of viscoelastic relaxation, defined by $\epsilon=0 \cdot 2,1,5$. The variations of $E_{R}, \phi_{R}$ and $E_{\text {int }}$ are plotted in figure 4. It is observed that a change in $\epsilon$ has almost no effect on the reflected energy except a little near the critical incident direction at $\theta_{o}=53^{\circ}$. The increase of $\epsilon$ is responded with a small decrease in phase shift of the reflected wave but only for incidence beyond the critical direction. The magnitude of interaction energy increases with the increase of anelasticity parameter $\epsilon$. The negative sign of interaction energy implies that the energy is directed towards the fluid-solid interface. With unchanged $E_{R}$, any change 

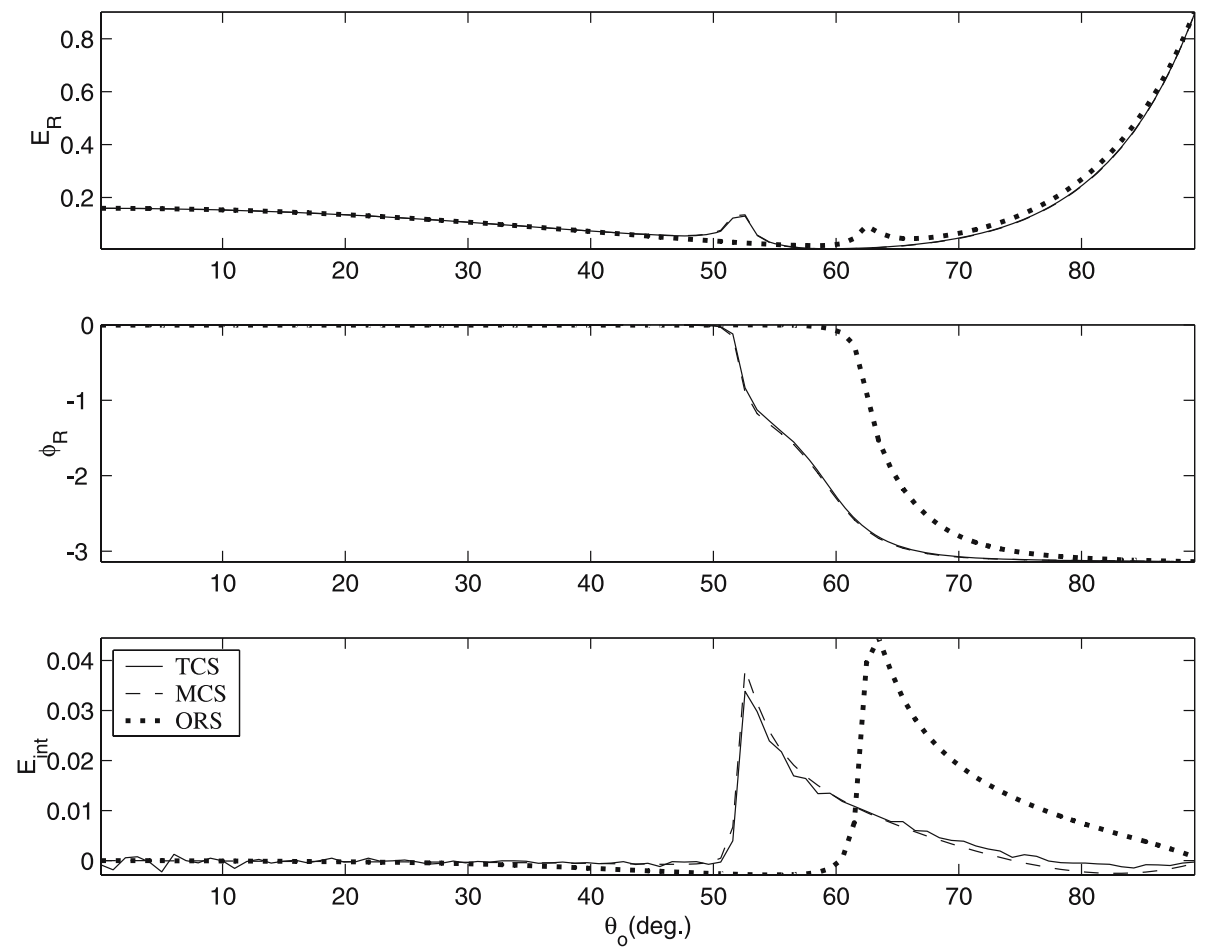

Figure 3. Variations of energy share $\left(E_{R}\right)$ and phase shift $\left(\phi_{R}\right)$ of reflected wave and interaction energy $\left(E_{\text {int }}\right)$ with incidence angle $\left(\theta_{o}\right)$ in $\phi_{o}=0.34 \pi$ plane, for different anisotropic symmetries, viz. triclinic (TCS), monoclinic (MCS), orthotropic (ORS), in alumina-epoxy and using the GL theory of thermoelasticity; $\left(\epsilon=1 ; \omega / 2 \pi=100 \mathrm{kHz} ; \tau_{0}=10^{-8} \mathrm{~s} ; T_{o}=300 \mathrm{~K} ; C_{e}=300 \mathrm{~J} / \mathrm{kgK} ; \chi_{o}=0.6 \mathrm{~W} / \mathrm{mK}\right.$; $\left.\alpha_{o}=0 \cdot 6 / 10^{4} \mathrm{~K}\right)$.

in interaction energy with $\epsilon$ implies an equally opposite change in the total energy of four refracted waves in ATV medium. Thus, the increase of negative interaction energy with almost same reflected energy implies stronger refracted waves with an increase in $\epsilon$. On the other hand, an increase of positive interaction energy should be seen as a reason to weaken the refracted waves.

\subsection{Specific heat}

Figure 5 presents the variations in $E_{R}, \phi_{R}$ and $E_{\text {int }}$ with incident direction $\theta_{o}$ for three different values of specific heat, given by $C_{e}=20,100,500 \mathrm{~J} / \mathrm{kgK}$. The critical angle for refracted $q P$-wave decreases with the decrease in $C_{e}$ of ATV medium. For the case of $C_{e}=20 \mathrm{~J} / \mathrm{kgK}$, this angle lowers down to $40^{\circ}$. Otherwise, ATV medium with a smaller specific heat coefficient may reflect a stronger acoustic wave into the fluid. This strength matters more around critical incident direction. An increase of $C_{e}$ reduces the phase shift of the reflected wave but for incidence beyond critical direction. A change in $C_{e}$ has a quite significant effect on the interaction energy of refracted waves. For incidence at $\theta_{o}>55^{\circ}$, the reflected wave is not affected with the increase of $C_{e}$. Then, the resulting increase in interaction energy implies a relatively weaker refracted waves in ATV solid of large specific heat. 

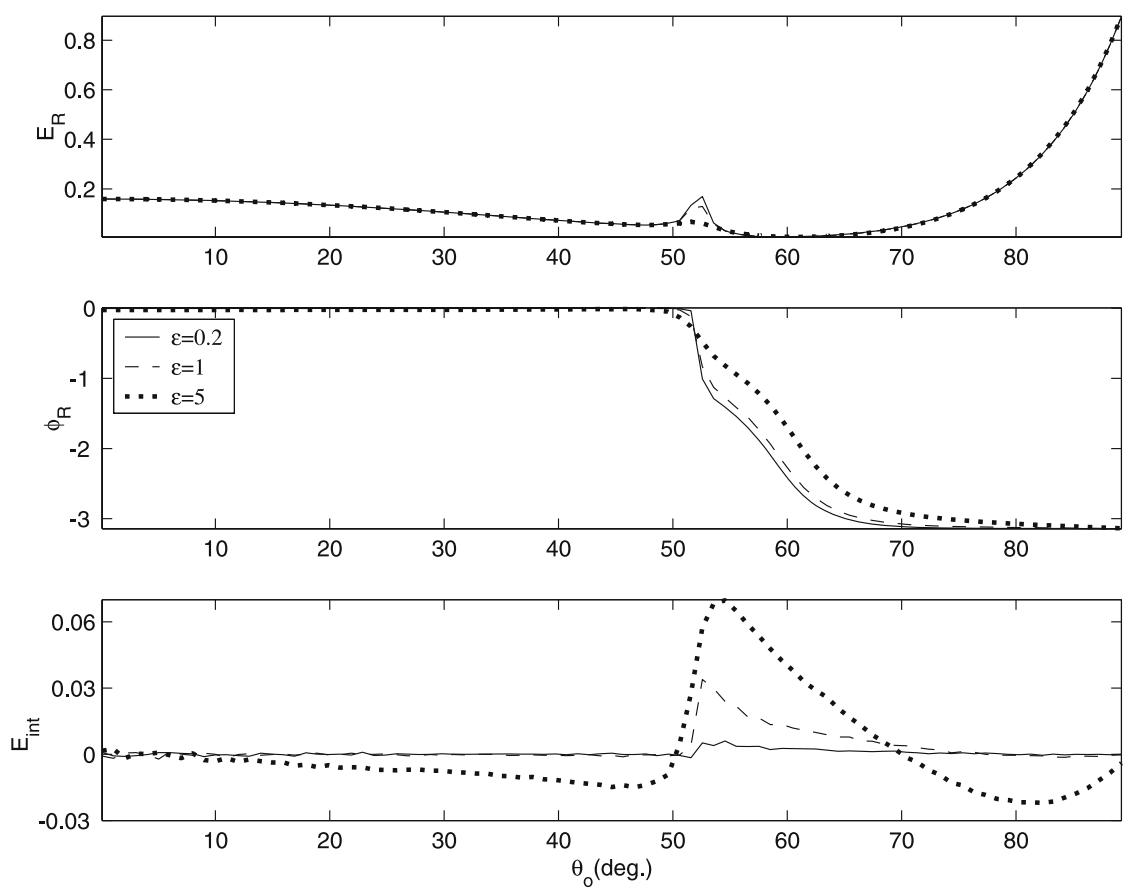

Figure 4. Same as figure 3, but for different values of anelasticity parameter $\epsilon$ and triclinic anisotropy (TCS) only.
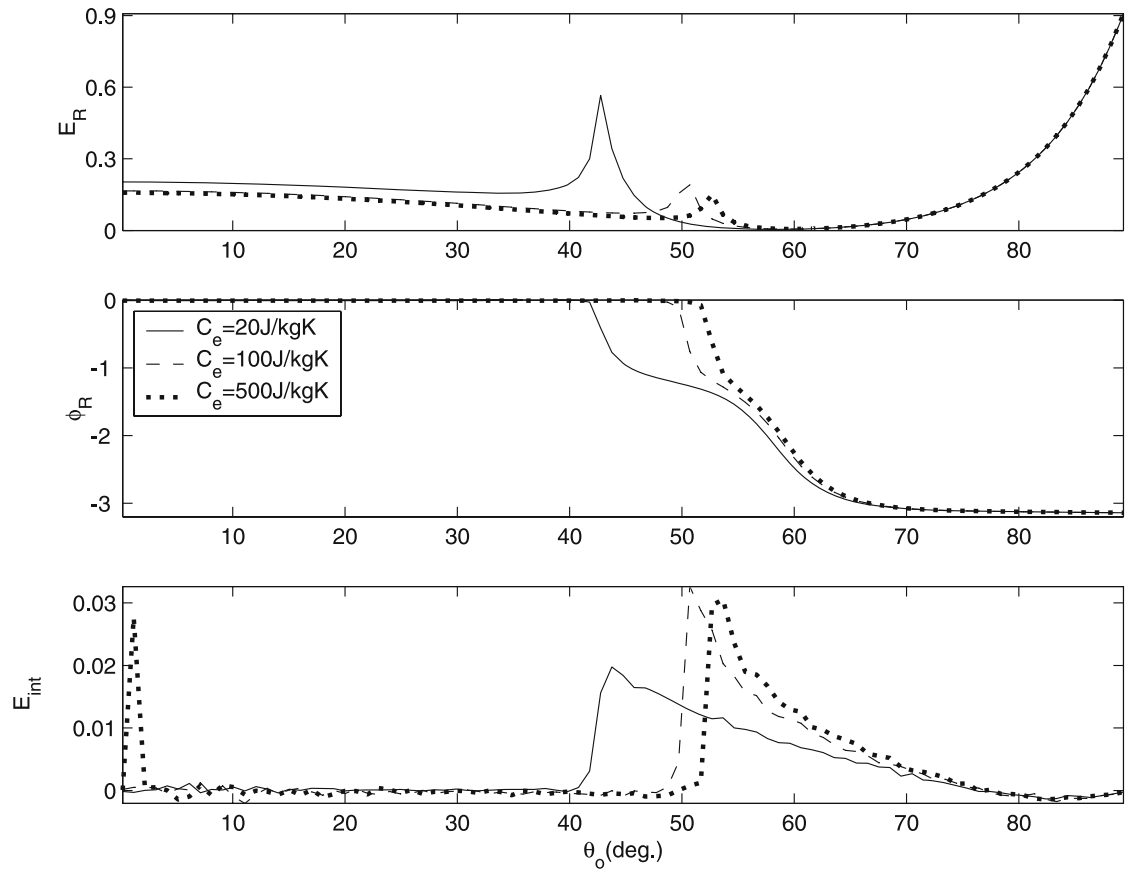

Figure 5. Same as figure 4, but for fixed anelasticity (i.e. $\epsilon=1$ ) and different values of specific heat $C_{e}$. 

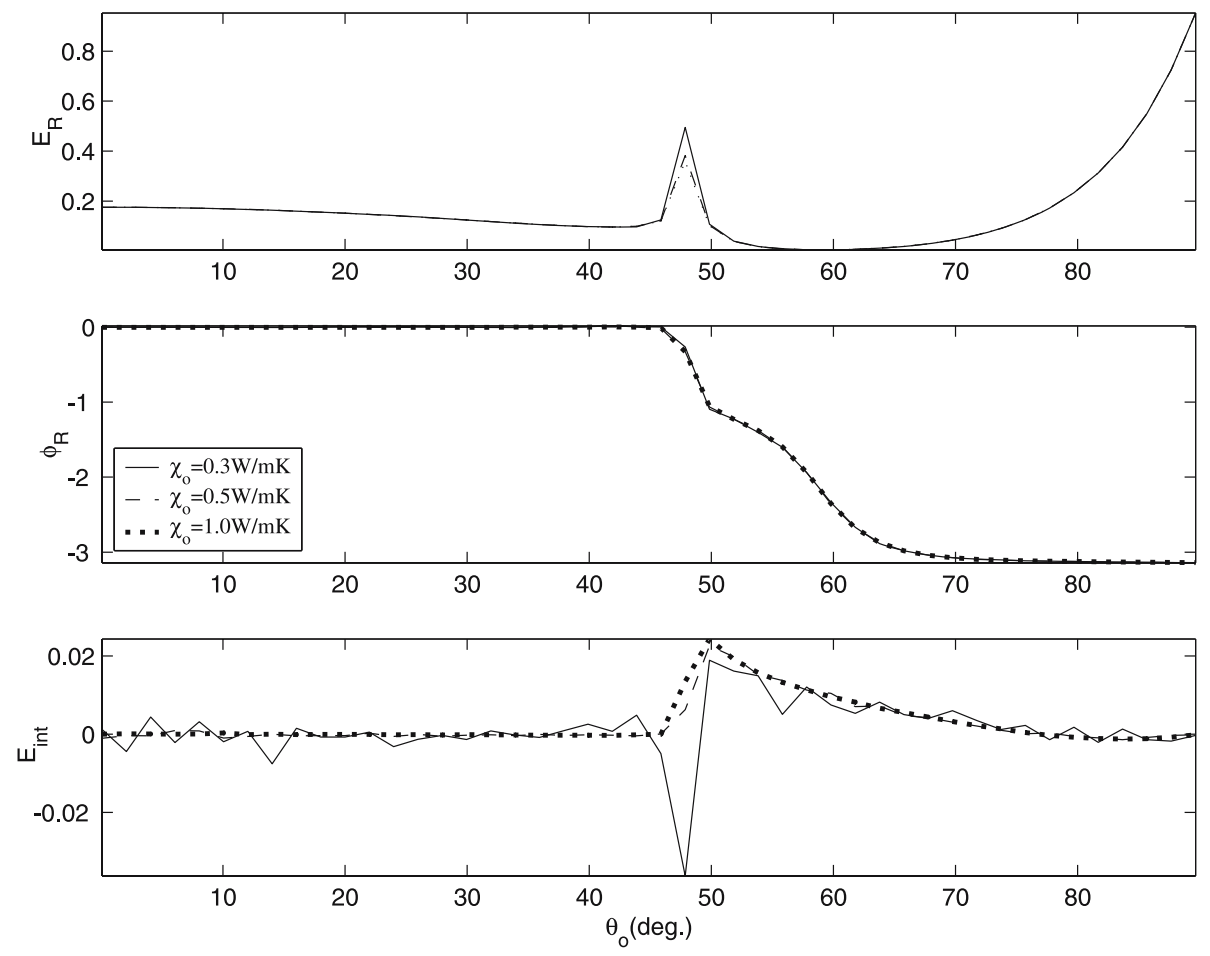

Figure 6. Same as figure 5, but for fixed specific heat (i.e. $C_{e}=100 \mathrm{~J} / \mathrm{kgK}$ ) and different values of thermal conductivity parameter $\chi_{o}$.

\subsection{Thermal conductivity}

The plots in figure 6 show the variations in $E_{R}, \phi_{R}$ and $E_{\text {int }}$ for the three values of thermal conductivity parameter $\chi_{o}=0 \cdot 3,0.5,1 \mathrm{~W} / \mathrm{mK}$. The effect of $\chi_{o}$ is evident only on $E_{\text {int }}$, which decreases significantly with an increase in $\chi_{o}$ from $0.3 \mathrm{~W} / \mathrm{mK}$. However, the increase in $\chi_{o}$ after $0.5 \mathrm{~W} / \mathrm{mK}$ is showing a much less effect. Phase shift of reflected wave does not change with the thermal conductivity of the underlying ATV solid bed. The reflected acoustic wave affects a bit with the change in thermal conductivity but only for incidence around the critical direction near $\theta_{o}=48^{\circ}$.

\subsection{Thermal expansion}

The figure 7 exhibits the effect of thermal expansion parameter $\alpha_{o}=0 \cdot 1,1,2$ (per $10^{4} \mathrm{~K}$ ) on the variations of $E_{R}, \phi_{R}$ and $E_{i n t}$ with incident direction. On comparison with the plots in figures 5 and 6 , the reflected wave seems to be more sensitive to thermoelastic coupling $\alpha_{i j}$ (or thermal expansion coefficient $\alpha_{o}$ ) than other thermal properties. However, sensitivity of $E_{R}$ is significant more for incidence below $55^{\circ}$. With an increase of $\alpha_{o}$, the reflected energy increases but the critical angle for refracted $q P$-wave decreases. For incidence beyond $55^{\circ}$, any change in $\alpha_{o}$ does not affect the reflected energy. For post-critical incidence, phase shift of reflected wave increases with the increase of $\alpha_{o}$. The change in $\alpha_{o}$ has a significant effect on interaction energy also but only for incidence at $\theta_{o}>35^{\circ}$. For incidence after $53^{\circ}$, an increase of $\alpha_{o}$ results in the decrease of interaction energy. This implies that stronger refracted waves propagate in an ATV medium of larger thermal expansion. 

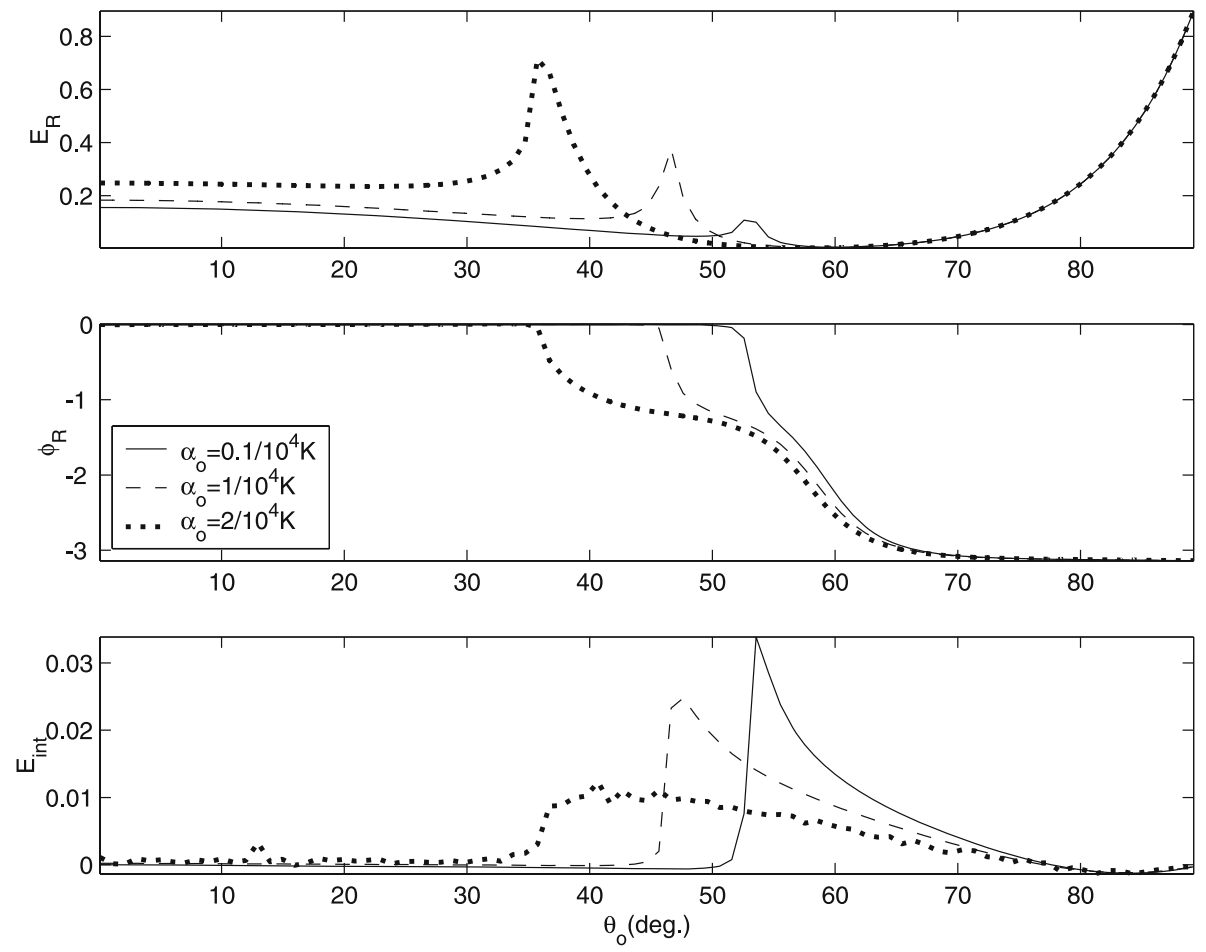

Figure 7. Same as figure 6 , but for fixed thermal conductivity (i.e. $\chi_{o}=5 \mathrm{~W} / \mathrm{mK}$ ) and different values of thermal expansion coefficient $\alpha_{0}$.

\subsection{Frequency, relaxation time, uniform temperature}

The reflected wave and interaction energy were found to be unaffected with any change in the value of wave frequency $(\omega / 2 \pi)$ between $1 \mathrm{kHz}$ to $1 \mathrm{GHz}$. This indicates a non-dispersive character for the reflected acoustic wave. The relaxation time $\left(\tau_{0}\right)$ and uniform temperature $\left(T_{o}\right)$ have only a negligible effect on the variations of $E_{R}, \phi_{R}$ and $E_{\text {int }}$ with incident direction. The energy partitions were computed for $\tau_{0}$ varying in $\left[10^{-12}, 10^{-4}\right] \mathrm{sec}$ and $T_{o}$ in $[250,500] \mathrm{K}$.

\section{Conclusions}

This study considers a mathematical model that relates the acoustic/seismic response of ATV medium to its physical/mechanical/thermal properties. ATV medium represents a general and realistic model for synthetic composites as well as crustal rocks. Analytical procedures derived in this study are used to characterize the polymer composites for sound absorbing properties. Numerical examples consider some particular models for the computation of energy partition among reflected and refracted waves. Energy conservation at isothermal interface is verified in each of the examples. Any generalization of results from the particular numerical model discussed in the previous section may not be safe. However, qualitative aspects of the discussion may lead to some observations that may be useful in simulation studies. Few of these observations may be concluded as follows. 
(i) Use of one or other theory of thermoelasticity may not predict much difference to the propagation characteristics of the acoustic waves reflected from isothermal solid base.

(ii) The azimuthal variations in the incident direction may not have much effect on the reflected wave. Hence, the propagation restricted to a fixed vertical plane may be able to explain the reflection response of isothermal solid surface.

(iii) There exist critical incident directions corresponding to the waves refracted to the ATV medium. For a refracted wave, this is the incident direction beyond which the wave propagates towards the boundary. It is observed that the reflected energy changes swiftly for incidence around such a critical direction. These critical directions may change with the changes in specific heat, thermal expansion, elastic properties and anisotropic symmetry of the ATV medium.

(iv) Energy share and phase shift of the reflected wave vary significantly for incidence around or after the critical incident directions of refracted waves. The reflected energy reduces to minimum after the critical directions of refracted waves but then increases swiftly to reach maximum at grazing incidence. Phase shift of the reflected wave also reaches its maximum value (i.e. $\pi$ ) near grazing incidence.

(v) A composite medium with higher specific heat may absorb more incident energy and reflect a comparatively weaker acoustic wave. However, such a behaviour may not be expected from a composite by increasing its viscoelastic relaxation.

(vi) For incidence below critical directions, total energy carried by the refracted waves in composite medium may not be affected with a change in its anisotropic symmetry or thermal conductivity. For such an incidence, a little interaction energy among the refracted waves implies a nearly non-attenuating behaviour of refracted waves. For incidence beyond critical directions, a decrease in the specific heat and/or an increase in linear thermal expansion of a composite medium may ensure a stronger refracted waves.

\section{References}

Ainslie M A, Burns P W 1995 Energy-conserving reflection and transmission coefficients for a solidsolid boundary. J. Acoust. Soc. Am. 98: 2836-2840

Biot M A 1956 Thermoelasticity and irreversible thermodynamics. J. Appl. Phys. 27: 240-253

Borcherdt R D 1977 Reflection and refraction of type-II S waves in elastic and inelastic media. Bull. Seism. Soc. Am. 43: 43-67

Carcione J M 2001 Wave fields in real media: Wave propagation in anisotropic anelastic and porous media (Amsterdam: Pergamon Press)

Dhaliwal R S, Sheriff H H 1980 Generalized thermoelasticity for anisotropic media. Quart. Appl. Maths. 38: 1-8

El-Karamany A S, Ezzat M A 2002 The uniqueness and reciprocity theorems for generalised thermoviscoelasticity with two relaxation times. Int. J. Engng. Sci. 40: 1275-1284

El-Karamany A S, Ezzat M A, Othman M I 2002 State-space approach to two-dimensional generalised thermo-viscoelasticity with two relaxation times. Int. J. Engng. Sci. 40: 1251-1274

Giorgi C, Naso M G, Vuk E 2001 Exponential stability in viscoelastic and elastic systems with thermal memory. Int. J. Differ. Eqs. Appl. 2: 55-91

Green A E, Lindsay K A 1972 Thermoelasticity. J. Elasticity 2: 1-7

Green A E, Naghadi P M 1993 Thermoelasticity without energy dissipation. J. Elasticity 31: 189-208

Helbig K 1994 Foundations of Anisotropy for Exploration Seismics (New York: Pergamon Press)

Hetnarski R B, Ignaczak J 1996 Soliton like waves in a low-temperature non-linear thermoelastic solid. Int. J. Engng. Sci. 34: 1767-1787 
Jeffereys H 1930 The thermodynamics of an elastic solid. Proc. Camb. Phil. Soc. 26: 101-106

Kovalenko A D, Karnaukhov V G 1972 A linearized theory of thermoviscoelasticity. Mech. Compos. Mater. 8: 194-199

Lord H W, Shulman Y 1967 The generalised dynamic theory of thermoelasticity. J. Mech. Phys. Solids 15: 299-309

Musgrave M J P 1970 Crystal Acoustics (San Francisco: Holden-Day)

Norwick A S, Berry B S 1972 Anelastic relaxation in crystalline solids (New York: Academic Press)

Paulino G H, Jin Z-H 2001 Correspondence principle in viscoelastic functionally graded materials. J. Appl. Mech. 68: 129-132

Puri P 1973 Plane waves in generalised thermoelasticity. Int. J. Engng. Sci. 11: 735-744

Sharma J N, Kumar V, Chand D 2003 Reflection of generalised thermoelastic waves from the boundary of a half-space. J. Thermal Stresses 26: 925-942

Sharma J N, Kumar V, Sud S P 2000 Plane harmonic waves in orthotropic thermoelastic materials. J. Acoust. Soc. Am. 107: 293-305

Sharma M D 2006 Wave propagation in anisotropic generalized thermoelastic medium. J. Thermal Stresses 29: 629-642

Sharma M D 2007 Propagation of inhomogeneous waves in a generalized thermoelastic anisotropic medium. J. Thermal Stresses 30: 678-691

Sharma M D 2008 Inhomogeneous waves at the boundary of a generalized thermoelastic anisotropic medium. Int. J. Solids \& Struct. 45: 1483-1496

Singh H, Sharma J N 1985 Generalised thermoelastic waves in transversely isotropic media. J. Acoust. Soc. Am. 77: 1046-1053

Wong C P, Bollampally R S 1999 Thermal conductivity elastic modulus and coefficient of thermal expansion of polymer composites filled with ceramic particles for electronic packaging. J. Appl. Poly. Sci. 74: 3396-3403 